\title{
Introduction to the Proceedings of CISCEM 2018 - the 4th Conference on In-Situ and Correlative Electron Microscopy
}

\author{
$\underline{\text { Niels de Jonge }^{1,2}}$, Kristian Mølhave $^{3}$ and Damien Alloyeau ${ }^{4}$ \\ ${ }^{1 .}$ INM - Leibniz Institute for New Materials, D-66123 Saarbrücken, Germany \\ 2. Department of Physics, Saarland University, D-66123 Saarbrücken, Germany \\ 3. Technical University of Denmark, Lyngby, Denmark \\ 4. CNRS / University Paris-Diderot, France
}

The Conference on In-Situ and Correlative Electron Microscopy (CISCEM) took place in Saarbrücken, Germany, for the fourth time [1], from October 10 to 12, 2018 (website: www.ciscem2018.de). CISCEM is about understanding nano- and atomic-scale processes under native and realistic conditions. In so-called in situ methods, observations and measurements are carried out in an environment similar to the real world, for example, in gases, in liquids, or at high temperatures.

The scope of CISCEM is to bring together an international and interdisciplinary group of scientists from the fields of biology, materials science, chemistry, and physics, to discuss future directions of in situ electron microscopy research. Progress in the field benefits from cross fertilization of expertise and ideas obtained using the different available methods for time-resolved imaging of physical, chemical and biological processes, thus inspiring development of new capabilities across disciplines: How can protein functions in cells be better investigated? What are the options for scientists to image soft materials in liquid by electron microscopy? What are the possibilities to analyze in processes involving nanomaterials (e.g., nanostructure formation and catalytic or electrochemical reactions)? How can modern data acquisition and data processing software algorithms help to increase the speed of timeresolved electron microscopy and/or reduce electron-beam effects? What are the opportunities and challenges of correlative approaches involving in situ TEM and other environmental techniques?

The highlight of the conference was the keynote lecture by Prof. Robert Sinclair on "High Resolution In Situ and Transmission Environmental Electron Microscopy of Material Reactions". The topics of the other sessions, lectures and posters covered 3 main themes:

- Session 1 on Light, electron and correlated microscopy of biological samples and session 2 on soft matter materials, where both sessions had contribution on how liquid phase TEM is being developed to provide insights in these fields.

- Session 3 on material reactions and session 4 on novel methods to study such processes and session 5 on the much-needed development of low dose imaging methods to avoid irradiation induced artefacts in the observations. There is a strong activity on innovations for in situ methods and data processing to allow ever lower dose imaging,

- Session 6 on nano-catalysts, session 7 on nanomaterial processes and session 8 on high temperature in-situ spectroscopy, show the recent progress in these important topics for in situ at high speed, high temperatures, and dynamic processes of nanomaterials also in liquids, where graphene membranes overall are increasingly explored for both biological and materials science imaging.

Invited speakers were:

- Prof. Nigel Browning, University of Liverpool, UK 
- Dr. Lucy Collinson, The Francis Crick Institute, UK

- Dr.-Ing. Tim Dahmen, German Research Center for Artificial Intelligence, Saarbrücken, Germany

- Dr. Indra Navina Dahmke, INM - Leibniz Institute for New Materials, Saarbrücken, Germany

- Dr. Radostin Danev, University of Tokyo, Japan

- Dr. Arnaud Demortière, CNRS / Université de Picardie Jules Verne, France

- Dr. Stig Helveg, Haldor Topsøe, Denmark

- Prof. R. J. Dwayne Miller, Max Planck Institute for the Structure and Dynamics of Matter, Germany

- Ass. Prof. Jaysen Nelayah, CNRS / Diderot University, Paris, France

- Ass. Prof. Joe Patterson, University of California, Irvine, CA, USA

- Prof. Erdmann Spiecker, Friedrich Alexander University Erlangen-Nuremberg, Germany

- Dr. Kinga A. Unocic, Oak Ridge National Laboratory, Oak Ridge, TN, USA

- Ass. Prof. Taylor Woehl, University of Maryland, USA

The scientific organization committee was:

- Prof. Niels de Jonge, INM-Leibniz Institute for New Materials, Saarbrücken, Germany

- Prof. Kristian Mølhave, DTU - Technical University of Denmark, Lyngby, Denmark

- Dr. Damien Alloyeau, CNRS - Université Paris-Diderot, France

Please note that the proceedings do not contain all abstracts of CISCEM because several authors preferred not to publish their abstract. The full conference program is available at http://www.ciscem2018.de

References:

[1] N de Jonge, K Mølhave and D Alloyeau, Microsc Microanal 22S5 (2016), p. 1.

[2] We thank Eduard Arzt for his support through INM and Christine Hartmann who took care of the entire conference organization.

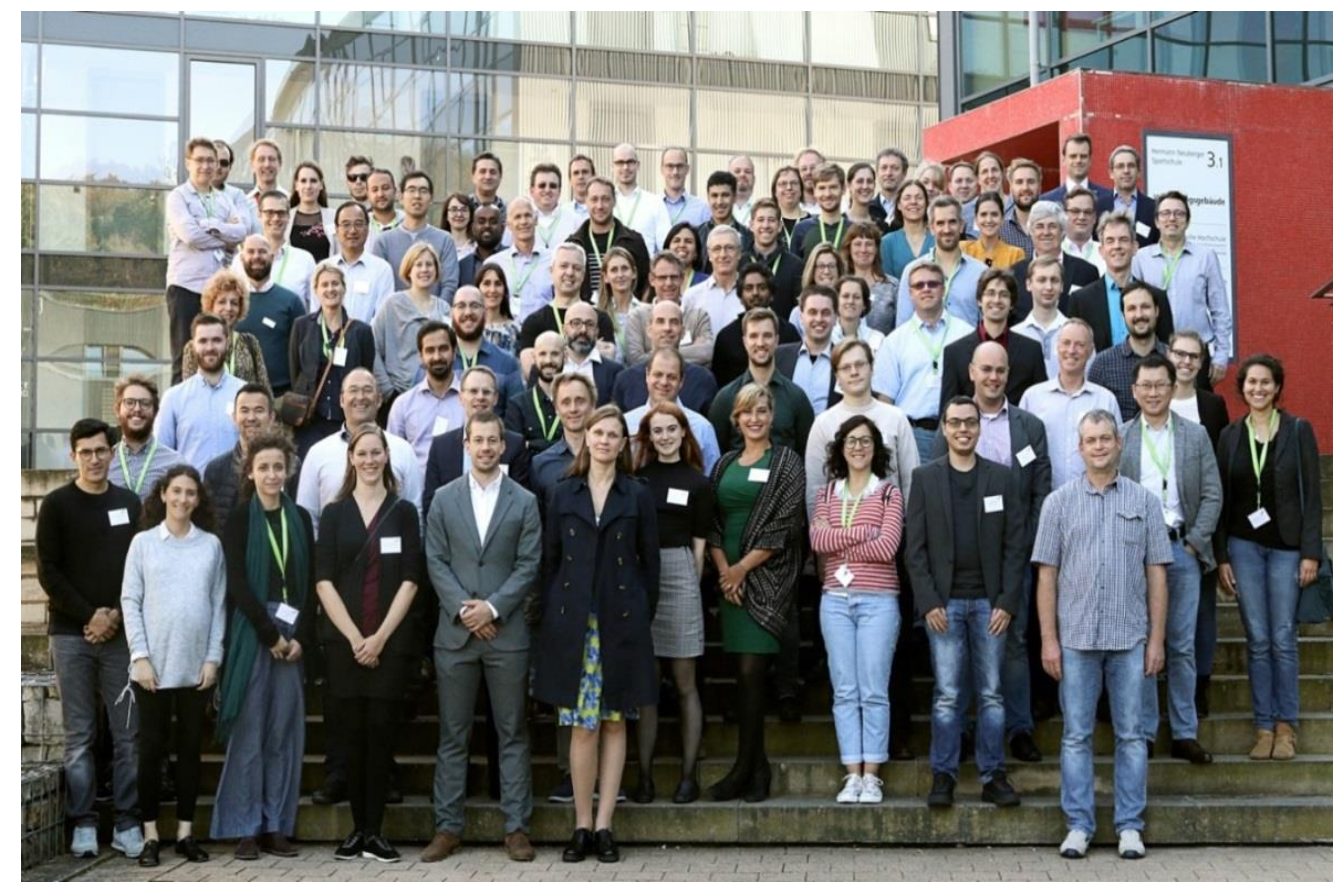

Figure 1: Group photo of CISCEM 2018. (Photo: Tabea Trampert) 